Conclusions Loss of BMPR-II predisposes to both an abnormal increase in IL-6 and IL-8 after stimulation with LPS and a dysfunctional response to these cytokines in PASMCs and mice. The mechanism for this involves loss of antioxidant function. This abnormal response may underlie the additional lung-specific trigger that promotes the development of PAH in patients with BMPR-II mutations, and may represent a target for future therapeutic interventions.

\section{S102 THE CROSSTALK OF PDE INHIBITOR WITH BMP SIGNALLING PATHWAY IN HUMAN PULMONARY ARTERIAL SMOOTH MUSCLE CELLS}

doi:10.1136/thoraxjnl-2011-201054b.102

J Yang, X Li, C Wu, N Morrell. University of Cambridge, Cambridge, UK

Rational Sildenafil, a potent PDE inhibitor, is an established treatment for PAH. However, the detailed mechanism of its effects on the proliferation of human pulmonary artery smooth muscle cells (hPASMCs) remains unclear.

Objective Because sidenafil is effective treatments for clinical $\mathrm{PAH}$, we hypothesised that these agents enhance Smad1/Id signalling through cGKI in hPASMCs.

Methods and Results Sildenafil alone has no effect on Smad1 phosphorylation and Id1 gene expression in hPASMCs, However in the presence of BMP4 Sidenafil indeed enhanced BMP-induced phosphorylation of Smad1/5 and Id1 expression in a cGMP/cGKIdependent manner in hPASMCs. The presumed mechanism is by elevation of intracellular cGKI activity which modulate smad1 phosphorylation and nuclear localisation. Knock down cGKI or use pharmalogical cGMP inhibitor abrogate the effect of Sildenafil on hPASMCs. Furthermore we confirm the rescued pSmad1 signal and elevated proliferation inhibitory effect in hPASMCs from familial pulmonary arterial hypertension patients by Sildenafil.

Conclusions Sildenafil enhance BMP/Smad through cGMP/cGKI pathway to modulate hPASMCs proliferation.

\section{Clinical studies in bronchiectasis S103 MICROBIAL COMMUNITY COMPOSITION IN THE LUNGS OF
PATIENTS WITH CYSTIC FIBROSIS AND NON-CF BRONCHIECTASIS}

doi:10.1136/thoraxjnl-2011-201054b.103

${ }^{1} \mathrm{G}$ G Einarsson, ${ }^{2} \mathrm{E}$ Klem, ${ }^{2} \mathrm{~A}$ A Fodor, ${ }^{1} \mathrm{~L}$ Wei, ${ }^{3} \mathrm{M}$ Drain, ${ }^{2} \mathrm{M} \mathrm{C}$ Wolfgang, ${ }^{3} \mathrm{~J} \mathrm{~S}$ Elborn, ${ }^{1} \mathrm{M}$ M Tunney. ${ }^{1} \mathrm{CF}$ and Airways Microbiology Research Group, Queen's University Belfast, Belfast, UK; ${ }^{2}$ University of North Carolina at Chapel Hill, Chapel Hill, North Carolina, USA; ${ }^{3}$ Centre for Infection and Immunity, Queen's University Belfast, Belfast, UK

Introduction and Aims Persistent bacterial infection is a major cause of morbidity and mortality in patients with both Cystic Fibrosis (CF) and non-CF Bronchiectasis (non-CFBX). Numerous studies have shown that CF and non-CFBX airways are colonised by a complex microbiota. However, many bacteria are difficult, if not impossible, to culture by conventional laboratory techniques. Therefore, molecular detection techniques offer a more comprehensive view of bacterial diversity within clinical specimens. The objective of this study was to characterise and compare bacterial diversity and relative abundance in patients with CF and non-CFBX during exacerbation and when clinically stable.

Methods Sputum samples were collected from CF ( $\mathrm{n}=50$ samples) and non-CFBX ( $\mathrm{n}=52$ samples) patients at the start and end of treatment for an infective exacerbation and when clinically stable.
Pyrosequencing was used to assess the microbial diversity and relative genera (or the closest possibly taxonomic order) abundance within the samples. Each sequence read was defined based on $3 \%$ difference.

Results High-throughput pyrosequencing allowed a sensitive and detailed examination of microbial community composition. Rich microbial communities were apparent within both CF (171 specieslevel phylotypes per genus) and non-CFBX airways (144 species-level phylotypes per genus). Relative species distribution within those two environments was considerably different; however, relatively few genera formed a core of microorganisms, representing approximately $90 \%$ of all sequences, which dominated both environments. Relative abundance based on observed operational taxonomic units demonstrated that the most abundant bacteria in CF were Pseudomonas (28\%), Burkholderia (22\%), Streptococcus (13\%), family Pseudomonadaceae (8\%) and Prevotella (6\%). In contrast, the most commonly detected operational taxonomic units in non-CFBX were Haemophilus (22\%), Streptococcus (14\%), other (unassigned taxa) (11\%), Pseudomonas (10\%), Veillonella (7\%) and Prevotella (6\%).

Conclusions These results suggest that distinctive microbial communities are associated with infection and/or colonisation in patients with both CF and non-CFBX. Although relatively high species richness was observed within the two environments, each was dominated by different core taxa. This suggests that differences in the lung environment of these two diseases may affect adaptability of the relevant bacterial taxa.

\section{S104 THE CULTURE MICROBIOME IN THE LUNGS OF PATIENTS WITH COPD}

doi:10.1136/thoraxjnl-2011-201054b.104

${ }^{1} \mathrm{G}$ G Einarsson, ${ }^{2} \mathrm{D}$ Comer, ${ }^{1} \mathrm{M} \mathrm{M}$ Tunney, ${ }^{2} \mathrm{~J} \mathrm{~S}$ Elborn. ${ }^{1} \mathrm{CF}$ and Airways Microbiology Research Group, Queen's University Belfast, Belfast, UK; ${ }^{2}$ Centre for Infection and Immunity, Belfast, UK

Introduction and Aims Previous studies have shown that the lungs of Cystic Fibrosis (CF) and bronchiectasis (BE, not caused by CF) patients are colonised by a range of aerobic and anaerobic bacteria. As bacteria are also implicated in the pathogenesis and progression of chronic obstructive pulmonary disease (COPD), this study aimed to determine the culture microbiome of the COPD airways.

Methods Samples were collected from 13 stable COPD patients during routine bronchoscopy. Bronchial washings were taken at a single location in the right middle lobe by flushing and removing $30 \mathrm{ml}$ of sterile saline. Samples were cultured under strict anaerobic conditions with bacteria detected by plating on both selective and non-selective agar media and quantified by total viable count (TVC). Identification of the cultured bacteria was performed by amplification and subsequent sequencing of the 16sRNA gene.

Results Mean $\mathrm{FEV}_{1}$ was 1.36 (range 0.84-2.26, mean per cent predicted $\mathrm{FEV}_{1}, 54 \%$ ), and the mean ratio $\left(\mathrm{FEV}_{1} / \mathrm{FVC}\right)$ was $51 \%$ Bacteria were detected in $12 / 13$ samples (92\%) with bacteria from the genera Streptococcus [12/13 samples, 92\%; mean (range) TVC $\left.9.62 \times 10^{5} \mathrm{cfu} / \mathrm{ml}\left(1.50 \times 10^{3}-1.42 \times 10^{7}\right)\right]$ and Haemophilus [4/13 samples, $31 \%$; mean (range) $6.40 \times 10^{4} \mathrm{cfu} / \mathrm{ml}$ $\left.\left(2.20 \times 10^{3}-1.60 \times 10^{5}\right)\right]$ most frequently detected. Anaerobic bacteria primarily from the genera Prevotella [8/13 samples, $62 \%$; mean (range) TVC $\left.1.12 \times 10^{4} \mathrm{cfu} / \mathrm{ml}\left(1.30 \times 10^{3}-4.20 \times 10^{4}\right)\right]$ and Veillonella $\left[5 / 13\right.$ samples, $38 \%$; mean (range) TVC $1.29 \times 10^{5} \mathrm{cfu} / \mathrm{ml}$ $\left.\left(4.20 \times 10^{3}-3.60 \times 10^{5}\right)\right]$ were also detected. Pseudomonas and Moraxella were not detected in any samples.

Conclusions Our results show that bacteria from the genera Streptococcus, Haemophilus, Prevotella and Veillonella are frequently present the airways of patients suffering from COPD. Taking account of the 
dilutional effect of the bronchial wash procedure and extrapolating to allow comparison with sputum data in our laboratory for CF and $\mathrm{BE}$, the relative load of bacteria from the genera Streptococcus, Prevotella and Veillonella is similar in these three airway diseases. The potential role of these bacteria in the progression and pathogenesis of COPD requires further investigation.

\section{S105 LONGITUDINAL MICROBIOLOGY OF ADULT NON-CF BRONCHIECTASIS}

doi:10.1136/thoraxjnl-2011-201054b.105

${ }^{1}$ W A Haja Sahabudeen, ${ }^{2} \mathrm{D}$ L Smith. ${ }^{1}$ University of Bristol, Bristol, UK; ${ }^{2}$ North Bristol Lung Centre, Bristol, UK

Introduction and Objectives A longitudinal microbiologic profile in adults with non-CF bronchiectasis (nCF-Br) is helpful in directing appropriate antibiotic therapy and may also have implications for prognosis. Information in this area is scarce with limited published data and small sample sizes of available studies. We have looked at longitudinal records from a group of nCF- $\mathrm{Br}$ patients attending a specialist clinic.

Methods All available sputum microbiology results for patients over a 5-year period were analysed.

Results 158 patients, average age 64.5 years (range 18-87 years), $58 \mathrm{M}: 100 \mathrm{~F}$, had 2 or more samples available for analysis. The majority (149 (94.3\%)) of these had a HRCT diagnosis. 23 (14.6\%) of the 158 patients cultured no organisms. Abstract S105 table 1 shows the distribution of organisms found colonising the remaining 141 patients. In a subset of 72 patients with $=6$ samples taken over an average period of 2.7 (SD 1.0) years, $2(2.8 \%)$ grew no organisms, 17 (23.6\%) grew a single organism, 21 (29.2\%) grew two, 14 (19.4\%) grew three, $18(25 \%)$ grew four or more different organisms on different occasions. Of the 806 samples analysed in this subset of patients, the majority grew single organisms, $83(10.3 \%)$ reported 2 or more isolates. Among 46 of these 72 in whom Pseudomonas spp. was isolated, the initial isolate was followed by persistent colonisation in 30 (65.2\%).

Conclusions The distribution of colonising pathogens among our larger patient group is similar to those found in other studies. We have shown a higher degree of variation in organisms found over time than has been previously shown. ${ }^{1}$

Abstract S105 Table 1 Distribution of organism colonisation in patients with non-CF bronchiectasis

\begin{tabular}{lc}
\hline Organism & $\begin{array}{l}\text { Colonisation rate } \\
\text { n (\%) }\end{array}$ \\
\hline Pseudomonas aeruginosa & $42(26.6)$ \\
Haemophilus influenzae & $22(13.9)$ \\
Coliforms (including Klebsiella spp., Serratia & $14(8.9)$ \\
spp., Proteus spp., Escherichia coli and & \\
Enterobacter clocae) & \\
Streptococcus pneumoniae & $11(7.0)$ \\
Aspergillus spp. & $8(5.1)$ \\
Moraxella catarrhalis & $6(3.8)$ \\
Staphylococcus aureus & $3(1.9)$ \\
MRSA & $2(1.3)$ \\
Others & $2(1.3)$ \\
No pathogens isolated & $23(14.6)$ \\
\hline
\end{tabular}

\section{REFERENCE}

1. Nicotra MB, Rivera M, Dale AM, et al. Clinical, pathophysiologic, and microbiologic characterisation of bronchiectasis in an ageing cohort. Chest 1995; 108:955-61.

\section{S106 A RANDOMISED DOUBLE BLIND 13 WEEK CROSSOVER TRIAL OF HYPERTONIC SALINE (HTS) (6\%) VS ISOTONIC SALINE (ITS) (0.9\%) IN PATIENTS WITH BRONCHIECTASIS}

doi:10.1136/thoraxjnl-2011-201054b.106

${ }^{1} \mathrm{~J} M$ Bradley, ${ }^{2} \mathrm{~K}$ Treacy, ${ }^{1} \mathrm{~B}$ O'Neill, ${ }^{1} \mathrm{~F}$ McCourt, ${ }^{3} \mathrm{~L}$ Green, ${ }^{1} \mathrm{E}$ Gardner, ${ }^{2} \mathrm{~J}$ S Elborn. ${ }^{1}$ University of Ulster, Newtownabbey, Northern Ireland; ${ }^{2}$ Queen's University, Belfast, Northern Ireland; ${ }^{3}$ Belfast HSC Trust, Belfast, Northern Ireland

Background Hypertonic saline is a hyperosmolar agent which increases mucociliary clearance in CF but there is little data available on its effectiveness in bronchiectasis.

Aim To determine the effectiveness of nebulised HTS (6\%) in comparison to ITS $(0.9 \%)$ on $24 \mathrm{~h}$ sputum production, lung function and patient related outcome measures in patients with stable bronchiectasis.

Design Proof of concept double blind cross-over study.

Methods Patients with clinical and CT diagnosis of bronchiectasis were randomised (double-blinded) to receive two consecutive 4week treatments of twice daily nebulised HTS (6\%) or ITS $(0.9 \%)$ each masked with quinine sulphate; with a 2 -week washout period between treatment cycles. The order of treatment was determined using concealed computerised randomised allocation performed by a statistician not involved in study conduct (EG).

Outcomes $24 \mathrm{~h}$ sputum volume/weight, lung function $\left(\mathrm{FEV}_{1}\right)$, cough (Leicester Cough Questionnaire, LCQ) and quality of life (Quality of Life Questionnaire-Bronchiectasis, QOL-B), and adverse events.

Results 19 patients (9M: 10F; Mean (SD): 61 (11) yrs; FEV 1,57 (23) $\%$ predicted; daily sputum weight 17 (17) grams) were recruited into this study. 13 patients completed both arms of the study. A patient and co-ordinator questionnaire indicated that the study design and the use of quinine sulphate was successful in achieving double blinding and masking HTS/ITS. HTS had a small to large effect (effect sizes $0.01-0.14$ ) on sputum and $\mathrm{FEV}_{1}, \mathrm{LCQ}$, and QOL-B. The overall trend was a benefit from HTS compared to ITS. There was a significant improvement in the physical domain of the LCQ $(-0.8$ (0.9), $\mathrm{p}=0.01)$ and the respiratory symptoms domain of the QOL-B $(-11.6(17.7), p=0.03)$ in the HTS cycle compared to the ITS. There was no differences in adverse events between the cycles.

Conclusion This study suggests that HTS may be effective in bronchiectasis. It provides data on study feasibility (study design and sample size) to justify a Phase 3 multicentre clinical trial to investigate the effectiveness of HTS in bronchiectasis.

\section{S107 THE INFLUENCE OF VIRAL SYMPTOMS IN INFECTIVE EXACERBATIONS OF NON-CYSTIC FIBROSIS BRONCHIECTASIS}

doi:10.1136/thoraxjnl-2011-201054b.107

${ }^{1} \mathrm{M}$ P Smith, ${ }^{2} \mathrm{C}$ J Doherty, ${ }^{2} \mathrm{~J}$ R W Govan, ${ }^{1} \mathrm{~K}$ Templeton, ${ }^{1} \mathrm{~A}$ T Hill. ${ }^{1}$ Royal Infirmary of Edinburgh, Edinburgh, UK; ${ }^{2}$ University of Edinburgh, Edinburgh, UK

Aim The aim of this study was to determine whether presence of viral symptoms influenced severity or outcome of exacerbations of bronchiectasis.

Methods Patients presenting to our outpatient service with exacerbations of bronchiectasis (defined as increasing cough, increasing sputum volume and worsening sputum purulence) requiring 2 weeks' oral antibiotic therapy were included. Patients reporting viral symptoms (defined as fever and any two of: unusual tiredness, headache, rhinorrhoea, sore throat, anorexia, myalgia, diarrhoea or vomiting) were compared with those who did not. The following outcomes were used to assess the exacerbation and response to 2 weeks' antibiotic therapy between the two groups: sputum 